\title{
Self-reported Dermatological Manifestations, Perceived Stress, and Body Image of Young Adults During the Greek Lockdown Caused by the COVID-19 Outbreak
}

\author{
Sofia Aperi ${ }^{1} \cdot$ Flora Koliouli $^{2}$ (ID
}

Accepted: 4 October 2021 / Published online: 26 October 2021

(C) Associação Brasileira de Psicologia 2021

\begin{abstract}
The aim of the study is to explore the perceived impact of social distancing measures due to COVID-19 on the manifestation of skin disorders and symptoms and their association with perceived stress and body image. For the purposes of this article, quantitative-driven mixed methods are used: the Greek versions of "Perceived Stress Scale" (Andreou et al., International Journal of Environmental Research and Public Health 8:3287-3298, 2011) to measure the stress levels among population, and "Multidimensional Body-Self Relations Questionnaire-Appearance Scales" (Argyrides and Kkeli, Psychological Reports 113:885-897, 2013) to examine body image satisfaction and assessment, along with open-ended questions are used. Questionnaires were distributed during the period of social distancing measures and 3 weeks following lockdown. Two hundred and fifty-three $(N=253)$ participants completed the survey: a control group of 164 participants without dermatological manifestations and a study group $(N=89)$ with dermatological manifestations.. Statistical analysis shows that adults with acne have a higher sense of self-efficacy in managing stressful situations than participants who have other skin disorders. Gender differences are observed as far as the perceived appearance is concerned. Thematic content analysis (Braun and Clarke, Qualitative Research in Psychology 3:77-101, 2006) shows that adults with skin symptoms associate their symptoms exacerbation with intrapersonal dimensions, such as stress and fear and environmental factors. The presence of skin disease is not necessarily associated with body satisfaction, body image, and cutaneous body image, although positively correlated with stress. The results are discussed and future recommendations are proposed.
\end{abstract}

Keywords Dermatological diseases $\cdot$ Perceived stress $\cdot$ Body image $\cdot$ Cutaneous body image $\cdot$ Acne; Social distancing

Flora Koliouli

fkoliouli@psych.uoa.gr

Extended author information available on the last page of the article 


\section{Introduction}

Human skin is considered to have both a biological dimension and psychological interaction with the environment (Anzieu, 1995). By using the term "skin manifestations," cutaneous signs, symptoms, or physiological effects of a nondermatological disorder are included. For example, in many systemic diseases, there are skin manifestations which are related (Sadeghzadeh-Bazargan et al., 2021). Skin diseases such as acne, eczema, and urticaria are some of the most common ones and they are characterized by red skin rashes which cause pruritus and occasionally pain and they are often triggered by stressful situations (Bolognia et al., 2014). Even though stress is an emotional and physical response to stressors (Boehncke \& Schön, 2015; Parisi et al., 2013; Schacter et al., 2012), perceived stress is the subjective evaluation of the stressor in a period of time or at a given moment. According to Lazarus and Folkman (1984), stress is the response to an event that could be threatening or harmful and requires psychological, physical, and/or behavioral actions for handling the stressor and its impact. Initially, a primary evaluation of a situation occurs, in order to assess the degree of stress and subsequently, on a secondary level, to evaluate the available means to handle the situation. The COVID-19 pandemic is considered a major stressor and a traumatic event with multiple consequences on the individual's psychological well-being (Taylor et al., 2020). High levels of stress and fear induced by the threat of the ongoing virus pandemic have adversely affected individuals' lives on a global scale (Zandifar \& Badrfam, 2020).

Dermatological diseases such as acne, urticaria, and eczema have an impact on body image perception. In a study conducted by Bowe et al. (2011), based on the participation of fifty-two $(N=52)$ acne patients, results revealed that those individuals experienced high levels of dissatisfaction and stress with respect to their appearance. Even though acne more commonly occurs among men, women show higher levels of dissatisfaction in comparison to men (Amr et al., 2014). Moreover, urticaria patients seem to have a poor body image and the skin disease has negative ramifications on their sexual life and self-esteem (Simsek et al., 2020). Other dermatological ramifications, such as dry skin, can result in stress (Saif et al., 2018).

There are many ways stress can physically affect the skin. A stress hormone known as cortisol may lead to an overproduction of sebum (oil) in skin glands, which causes acne breakouts. Stress has repercussions on the immune system, causes a more reactive and sensitive skin, and triggers rashes, hives, and redness. Stress exacerbates existing inflammatory skin conditions like eczema, psoriasis, and rosacea, leading to flare ups. Stress may precipitate nervousness or anxiety, urge an individual to pick off scabs or acne, or ultimately lead to scratching one's skin until it develops into redness or breaks (Hosoi, 2006).

Body image is a part of the self-image (Petersen et al., 1984) and refers to the experience of weight, shape, and functionality of one's body. Body image has multiple definitions and it is consists of different facets. One definition of body image that includes a number of these dimensions is the following: "the 
systematic, cognitive, affective, conscious, and unconscious representation that people have concerning their bodies during their biological development and throughout their social relationships" (Roy \& Payette, 2012, p. 506). Metaanalysis research (Cleary et al., 2020) suggests several tools that assess and evaluate body image dimensions that have the tendency to focus on dissatisfaction and internalization of sociocultural ideas of attractiveness (Cash et al., 2005; Thompson \& Schaefer, 2019; Thompson, 2004). Grogan (2017) defines body image as the perceptions, thoughts, and feelings about one's body. As it appears, body image is a complex balance between perceptions and satisfaction, which is quite predominant in the Western societies, especially in terms of preoccupation with physical beauty and ideal body types (Partridge, 1993).

Based on the same notion, healthy skin is also recognized as a beauty standard and a trait of physical appearance (Mikołajczyk et al., 2017). However, a dermatological disorder, due to its visibility, could induce a certain aversion and fear to others because it may insinuate signs of a potential contagiousness, bad hygiene, and self-neglect (Cash, Morrow, Hrabosky, \& Perry, 2004). As far as the skin is concerned, the term of the cutaneous body image emerges and refers to the perception of the skin, hair, and nails (Gupta et al., 2004). This image could be distorted, when perception and interpersonal experiences diverge, in similar ways that the overall body image may be affected. Investigating the relationship between body image and dermatological manifestations could provide compelling results because one's skin is visible and directly intertwined with body image as well as beauty in Western societies.

To our knowledge, current research in Greece investigates skin manifestations related to systemic diseases and it is primarily conducted in a medical context (Stefanaki et al., 2002; Georgiadis et al., 2021). Thus, there is a gap in the literature that explores the dermatological manifestations related to perceived stressors and how this affects their cutaneous body image. Moreover, the pandemic outbreak imposed unprecedented circumstances of social distancing and the lack of academic research on this contemporary subject bolsters the significance of this investigation. The emergence of COVID-19, intensified by its rapid growth and spread, has exacerbated anxiety on a global scale and even stimulated an increase in mental health disorder cases (Salari et al., 2020; Taylor et al., 2008).

The objective of this study is to explore the perceived impact of social distancing measures aiming to control the spread of the virus in the self-reported manifestations of skin disorders, associated symptoms, and their correlation with perceived stress and body image.

According to the existing literature, higher levels of perceived stress are anticipated in the group engendering dermatological manifestations than the control group; lower levels of body image satisfaction are also expected to be found (Cash, 2004; Mikołajczyk et al., 2017).

In addition to this, we expect that women exhibit higher levels of perceived stress compared to men (Andreou et al., 2011) and lower levels of cutaneous body image satisfaction (Gupta \& Gupta, 2013).

Furthermore, lower body image satisfaction results are expected to be seen among females as opposed to men (Argyrides \& Kkeli, 2013). 
Finally, we aim to explore the perceived stressors encouraged by COVID-19, which might induce or trigger dermatological symptoms associated with a skin disease.

\section{Methods}

\section{Participants}

Participants were chosen through a convenience sample obtained by posting on social media. Participation criteria included whether individuals (a) spoke Greek, (b) had between 18 and 45 years of age, and (c) could access online social groups. Two hundred and fifty-five participants answered the survey; two of them were excluded because they were under 18 years old. The final sample consisted of $N=253$ participants, 167 women [66\%], 85 men [33.6\%], and one non-binary. Their mean age is $M=25.5$ years old ( $\mathrm{SD}=6.2$ years). More specifically, mean age for women in our sample is $M=25.61$ years old ( $\mathrm{SD}=5.91$ years) and for men it is $M=25.72$ years old $(\mathrm{SD}=6.82$ years $)$.

As far as marital status is concerned ( $c f$. Table 1), 52.2\% of the participants are single $(N=132)$ and $31.2 \%(N=79)$ are in a relationship. Married participants represent $7.9 \%(N=20)$. Moreover, a meager $0.8 \%$ of the sample $(n=2)$ is divorced and none is widowed.

Table 1 Participants' sociodemographic data

\begin{tabular}{lll}
\hline Demographics & $\%$ & $N$ \\
\hline Marital status & & \\
Single & 52.2 & 132 \\
Partnership & 31.2 & 79 \\
Married & 7.9 & 20 \\
Living with a partner & 7.9 & 20 \\
Divorced & 0.8 & 2 \\
Occupation & & \\
Students & 51.4 & 130 \\
Employees & 31.2 & 79 \\
Working students & 9.9 & 25 \\
Unemployed & 5.5 & 14 \\
Dermatological manifestations and/or diseases & \\
Acne & 15 & 44 \\
Eczema & 9.9 & 26 \\
Urticaria & 2 & 5 \\
Psoriasis & 1.2 & 3 \\
Neurodermatitis & 1.2 & 3 \\
Vitiligo & 0.8 & 2 \\
Multiple & 1.6 & 4 \\
Other & 0.8 & 2 \\
\hline & &
\end{tabular}


The majority of our sample were students $[51.4 \%(N=130)]$, most of the remaining participants were employees [31.2\%, N=79], and only $25(9.9 \%)$ did not do not belong to either one of these two categories. $64.8 \%(N=164)$ of the sample has no dermatological disease in contrast to $35.2 \%(N=89)$. Out of this $35.2 \%, 15 \%$ has been diagnosed with acne $(N=38)$ and $9.9 \%(N=25)$ with atopic dermatitis (eczema). Besides the diagnosed dermatological disease, several participants stated that they suffer from dermatological symptoms $(N=125)$, such as dry skin $(25.7 \%)$ $(N=65)$. Most of the manifestations with $32.8 \%(N=83)$ refer to face and arms with $24.5 \%(N=62)$.

\section{Material and Methods}

Socio-demographic Data The data was obtained via a self-report questionnaire and factors such as gender, age of the participants, marital status, level of education, occupation, and dermatological manifestations such as acne, eczema, and urticaria are taken into consideration. In this study, participants are asked to perform an evaluation of their own presence and intensity of skin manifestations.

Perceived Stress Scale (PSS) The Perceived Stress Scale (PSS; Cohen et al., 1983) is one of the most popular tools for measuring psychological stress. It is a selfreported questionnaire that was designed to measure "the degree to which individuals appraise situations in their lives as stressful" (Cohen et al., 1983, p. 385). The PSS items evaluate the degree to which individuals believe their life has been unpredictable, uncontrollable, and overloaded during the previous month. The assessed items are of general nature rather than focusing on specific events or experiences. The original instrument is a 14-item scale (PSS-14) (Cohen et al., 1983) including seven positive items (e.g., "In the last month, how often have you felt that you were on top of things?") and seven negative ones (e.g., "In the last month, how often have you found that you could not cope with all the things that you had to do?") rated on a 5-point Likert scale, going from $0=$ never to $4=$ very often. Cronbach's alpha of the PSS-14 is equal to $>0.70$, showing an acceptable internal consistence.

Multidimensional Body-Self Relations Questionnaire (MBSRQ) The MBSRQ measures a wide variety of attitudes towards body image (Muth \& Cash, 1997; Cash, 2002 in Argyrides \& Kkeli, 2013). There are two versions of the MBSRQ. The first edition of the instrument is a 69-item scale with 7 subscales, that reflect the dimensions of "Appearance Evaluation" and "Appearance Orientation" and 5 more subscales relating to appearance, physical condition, and health/illness. The Greek version which was used in our study had three further subscales: "Body areas satisfaction," "Overweight preoccupation," and "Self-classified weight" (Cash, 2002 in Argyrides \& Kkeli, 2013). Respondents are asked to answer on a 5-point Likert scale, going from $1=$ very dissatisfied to $5=$ very satisfied. More specifically, the "Appearance Evaluation" dimension corresponds to one's self-perception of attractiveness and appearance satisfaction and it is consisted of seven items (e.g., "Have a body that is sexually appealing"). "Appearance Orientation" estimates the 
degree of investment in someone's appearance and consists of twelve items (e.g., "Before going out, I notice how I look"). As far as the "Overweight Preoccupation" is concerned, it defines the perceived stress in terms of weight vigilance, diet, and nutritional restraint and it is measured through four items (e.g., "I am always on diet to lose weight"). "Self-classified weight" refers to weight perception and definition and it is evaluated through two items on a 5-Likert scale (e.g., "I think I am: a) very skinny, b) skinny, c) of normal weight, d) overweight, e)obese"; "Looking at me, most people would think that I am: a) very skinny, b) skinny, c) of normal weight, d) overweight, e)obese"). Finally, "Body Areas Satisfaction" includes eight items and investigates satisfaction or dissatisfaction with certain body areas (e.g., "Height"; "Thighs"). Cronbach's alpha of the MBSRQ was $>0.80$, showing a high internal consistency.

Cutaneous Body Image For the purposes of this study, the Cutaneous Body Image (Gupta et al., 2004) was adapted in order to investigate the skin, hair, and nails perception. Four items were adopted for our survey: "I am satisfied with the appearance of my fingernails," "I am satisfied with the appearance of my toenails," "I am satisfied with my hair," "I like my complexion or overall color of my skin." In order to assess the internal consistency of this scale in Greek, we proceeded to an exploratory factor analysis using VARIMAX rotation. The Bartlett test of Sphericity was used $(p=0.000)$ whereas the suitability of the data was checked by using the Kaiser-Meyer-Olkin Measure criterion $(\mathrm{KMO}=0.70)$. Cronbach's alpha value was equal to 0.69 , showing an acceptable internal consistency. Exploratory factor analysis indicated only one factor, the «cutaneous body image» with eigenvalue $=2079$ and $52 \%$ of the total variance is explained.

Open-ended questions seek to explore the perceived changes of dermatological manifestations during the last month of the lockdown attributed to the COVID-19 outbreak, which will be well remembered as a period of social distancing ("In your opinion, in which way (s) social distancing has affected your acne / atopic dermatitis (eczema)/ urticaria/ situation or the manifestation of other dermatological symptoms?").

Procedure

An online survey, created on Google Forms, was posted on social media in several groups. All participants were informed of the study's objectives and they completed a consent sheet beforehand. All information is confidential and anonymized.

We used the Greek versions of the PSS scale and MBSRQ scale. In translating the CBI scale to Greek, the recommendations of Beaton et al. (2000) and Stalikas et al., 2012 were followed; a forward and backward translation method was used. In this process, the English version of the CBI was translated to Greek by a professional translator and then a different professional translator back-translated the Greek version to English. Both translations were evaluated by the research team.

\section{Data Analysis}

Quantitative dominant [or quantitatively driven] mixed methods research is the type of mixed research in which one relies on a quantitative, post-positivist view of the 
research process, while concurrently recognizing that the addition of qualitative data and approaches are likely to benefit most research projects (Johnson et al., 2007, p. 124 in Schoonenboom \& Johnson, 2017).

$T$-test analysis was performed to investigate the gender differences in all variables. Bivariate correlation analyses were performed to examine the relationship between stress, body image, and cutaneous body image. All tests were 2-tailed, and the significance level was set at 0.05 . Hierarchical regression analysis was performed in order to assess the impact of stress and body image on cutaneous body image. Furthermore, analysis of variance (ANOVA) was performed when required in order to analyze the differences among group means in our sample. All statistical procedures were completed using the SPSS 18.0 Statistical Package for Social Science. As far as the qualitative analysis is concerned, we proceeded according to the six phases proposed by Braun and Clarke (2006): becoming more familiar with the data; generating initial codes; searching for themes; reviewing themes; defining and naming themes. The final phase involves the production of the report.

\section{Results}

We performed the Shapiro-ilk test $(=0.207)$ in order to determine the normality of the sample. More specifically, for the PSS scale, skewness for women is equal to $z=0.351(\mathrm{SE}=0.188)$, and kyrtosis is $z=0.524(\mathrm{SE}=0.374)$. As far as men are concerned, skewness is equal to $z=0.113(\mathrm{SE}=0.261$ ) and kyrtosis is equal to $z=-0.414(\mathrm{SE}=0.517)$. So we proceeded to parametric statistical analysis.

Firstly, descriptive results are presented followed by inferential statistical analysis. As presented in Table 2, our sample exhibits moderate levels of stress $(M=25.52$; $\mathrm{SD}=6.07)$, with a prevalence of the aspect of sense of helplessness $(M=16.22$; $\mathrm{SD}=5.30)$. The evaluation of the cutaneous body image is high $(M=11.94$; $\mathrm{SD}=2.45$ ). Among MBSRQ dimensions, body areas satisfaction is the highest

Table 2 Descriptive statistical analysis and Cronbach's alpha for total sample $N=253$

\begin{tabular}{lllllll}
\hline & $\#$ items & M & SD & Min & Max & Alpha \\
\hline Perceived Stress Scale & 14 & 25.52 & 6.07 & 0 & 55 & 0.60 \\
Sense of helplessness & 7 & 16.22 & 5.30 & 0 & 27 & 0.60 \\
Sense of self-efficacy & 7 & 9.31 & 5.10 & 0 & 28 & 0.65 \\
Multidimensional Body-Self & 34 & 111.73 & 12.87 & 71 & 144 & 0.80 \\
$\quad$ Relations Questionnaire & & & & & & \\
Appearance evaluation & 7 & 24.33 & 5.79 & 7 & 35 & 0.90 \\
Appearance orientation & 12 & 40.31 & 6.02 & 24 & 58 & 0.80 \\
Overweight preoccupation & 4 & 9.69 & 3.74 & 4 & 20 & 0.73 \\
Self-classified weight & 2 & 6.02 & 1.67 & 2 & 10 & 0.91 \\
Body areas satisfaction & 8 & 31.36 & 6.02 & 15 & 44 & 0.81 \\
Cutaneous Body Image & 4 & 11.94 & 2.45 & 5 & 16 & 0.69 \\
\hline
\end{tabular}


Table 3 Means, standard deviations, and $t$-test of Perceived Stress Scale in dermatological disease

\begin{tabular}{|c|c|c|c|c|c|}
\hline \multicolumn{6}{|l|}{ Dermatological disease } \\
\hline & \multicolumn{2}{|l|}{ No } & \multicolumn{3}{|l|}{ Yes } \\
\hline & $M$ & SD & $M$ & SD & $t$-test $(\mathrm{df}=251)$ \\
\hline Perceived Stress Total & 25.10 & 6.28 & 26.30 & 5.62 & -1.50 \\
\hline Helplessness & 16.47 & 5.13 & 15.75 & 5.61 & 1.02 \\
\hline Self-efficacy & 8.63 & 5.03 & 10.55 & 5.02 & $-2.89 * *$ \\
\hline
\end{tabular}

Note. ${ }^{*} p<0.05 ; * * p<0.01 ; * * * p<0.001$.

(comparing items and means) $(M=31.36 ; \mathrm{SD}=2.45)$ along with appearance evaluation $(M=24.33 ; \mathrm{SD}=5.79)$ and appearance orientation $(M=40.31 ; \mathrm{SD}=6.02)$.

To test the first hypothesis, we performed an independent samples $t$-test in order to measure the differences between the group presenting skin manifestations and the control group.

There was a significant difference in the scores for self-efficacy for individuals presenting skin manifestations $(M=10.55, \mathrm{SD}=5.02)$ and those who do not $(M=8.63, \mathrm{SD}=5.03) ; t(251)=-2.89, p=0.004$. These results suggest that the presence of dermatological manifestations impacts the sense of self-efficacy. Specifically, our results suggest that individuals with dermatological manifestations exhibit higher sense of self-efficacy (see Table 3).

Then, we proceeded to an ANOVA analysis to better nuance the type of skin manifestations that may influence the levels of self-efficacy. As demonstrated at Table 4, a statistically significant difference can be observed in the self-efficacy subscale between those suffering from acne and individuals not suffering from any skin diseases.

A one-way between-subjects ANOVA was conducted to compare the effect of perceived stress on individuals with no dermatological diseases, with acne, and with eczema. There was a significant effect of the sense of self-efficacy the $p<0.05$ level for the three conditions $[F(2,225)=6.37, p=0.001]$. Post hoc comparisons using the Scheffé test indicated that the mean score for the individuals with acne condition $(M=11.52, \mathrm{SD}=4.96)$ was significantly different than those who had not any dermatological conditions $(M=8.53, \mathrm{SD}=5.00)$. However, individuals with eczema condition $(M=10.27, \mathrm{SD}=4.93)$ did not significantly differ from the other two groups.

We proceeded to an independent samples $t$-test in order to measure the differences between the group presenting skin manifestations and the control group in

Table 4 Means and variance analysis of Perceived Stress Scale on types of dermatological manifestations

\begin{tabular}{lllll}
\hline & None & Acne & Eczema & \\
& $M$ & $M$ & $\mathrm{M}$ & $\mathrm{F}(\mathrm{df}=2 ; 225)$ \\
\hline Perceived Stress Total & $25.05_{\alpha}$ & $26.56_{\alpha}$ & $26.69_{\alpha}$ & 1.63 \\
Helplessness & $16.52_{\alpha}$ & $15.05_{\alpha}$ & $16.42_{\alpha}$ & 1.39 \\
Self- efficacy & $8.53_{\alpha}$ & $11.52_{\beta}$ & $10.27_{\alpha}$ & $6.37 * * *$ \\
\hline
\end{tabular}

Note. ${ }^{*} p<0.05 ; * * p<0.01 ; * * * p<0.001$. 
terms of body image satisfaction but there was no statistical difference in any of the measures variables (appearance evaluation, appearance orientation, body areas satisfaction, self-classified weight, and overweight preoccupation).

Our second hypothesis concerned the gender differences in terms of perceived stress and body image. As we observe, women are more appearance oriented $(M=41.09, \mathrm{SD}=6.54)$ than men $(M=38.97, \mathrm{SD}=6.40),[t(250)=2.44, p=0.01]$. Moreover, women are less satisfied with their cutaneous body image $(M=11.62$, $\mathrm{SD}=2.24)$ than men $(M=12.58, \mathrm{SD}=2.50),[t(251)=2.95, p=0.003]$.

Before performing the hierarchical regression analysis, we proceeded to a bivariate correlation among the dependent variables in order to assess the impact they would have on one another. As illustrated below ( $c f$. Table 5), higher levels of a sense of helplessness reduce the sense of self-efficacy and the appearance orientation $(r=-0.16 ; p=0.009)$. The more helpless feels the individual, the less they will be oriented to the way they look. Moreover, higher levels of cutaneous body image satisfaction are associated with high levels of appearance orientation $(r=0.39$; $p=0.000)$, and different body areas satisfaction $(r=0.52 ; p=0.000)$. The more satisfied the person is with its cutaneous body image, appearance evaluation, and body areas satisfaction increase. On the contrary, self-classified weight is negatively associated with appearance evaluation $(r=-0.47, p=0.000)$, and body areas satisfaction $(r=-0.43, p=0.000)$. However, higher levels of self-classified weight lead to higher levels of overweight preoccupation $(r=0.47, p=0.000)$. As far as appearance evaluation is concerned, it is positively linked to body areas satisfaction $(r=0.80$, $p=0.000)$, but it reduces overweight preoccupation $(r=-0.29, p=0.000)$. Along the same lines, appearance orientation increases overweight preoccupation $(r=0.30$, $p=0.000)$.

Given that bivariate correlation was high among dependent variables and since our last hypothesis inquiries the impact of perceived stress and body image on cutaneous body image, we proceeded to a hierarchical regression analysis in order to determine whether CBI could be predicted by the aforementioned variables. When perceived stress, it does not moderate the impact on the cutaneous body image. Nevertheless, when body image is inserted, we can observe that it has a significant

Table 5 Bivariate correlations of dependent variables

\begin{tabular}{|c|c|c|c|c|c|c|c|c|}
\hline Dependent variables & 1 & 2 & 3 & 4 & 5 & 6 & 7 & 8 \\
\hline 1. Sense of helplessness & 1.00 & & & & & & & \\
\hline 2. Sense of Self-efficacy & $-0.319 * * *$ & 1.00 & & & & & & \\
\hline $\begin{array}{l}\text { 3. Cutaneous Body } \\
\text { image }\end{array}$ & 0.00 & -0.06 & 1.00 & & & & & \\
\hline 4. Self-classified weight & -0.01 & 0.12 & -0.10 & 1.00 & & & & \\
\hline 5. Appearance evaluation & 0.06 & -0.01 & $0.39 * * *$ & $-0.47 * * *$ & 1.00 & & & \\
\hline 6. Appearance orientation & $-0.16^{* *}$ & 0.01 & 0.02 & -0.11 & 0.05 & 1.00 & & \\
\hline 7. Body areas satisfaction & 0.01 & -0.02 & $0.52 * * *$ & $-0.43 * * *$ & $0.80^{* * *}$ & 0.01 & 1.00 & \\
\hline 8. Overweight preoccupation & -0.06 & $0.12 *$ & $-0.13^{*}$ & $0.47 * * *$ & $-0.29 * * *$ & $0.30 * * *$ & $-0.33 * *$ & 1.00 \\
\hline
\end{tabular}

Note. ${ }^{*} p<0.05 ; * * p<0.01 ; * * * p<0.001$ 
Table 6 Summary of hierarchical regression analysis for variables predicting $\mathrm{CBI}$ $(N=253)$

\begin{tabular}{lllllll}
\hline \multicolumn{2}{l}{ Model 1 } & \multicolumn{5}{l}{ Model 2 } \\
\hline Variable & $B$ & SEB & $\beta$ & $B$ & SEB & $\beta$ \\
Perceived stress & -0.20 & 0.02 & -0.05 & -0.01 & 0.02 & -0.04 \\
Body image & & & & $0.07 * * *$ & 0.01 & 0.37 \\
$R^{2}$ & & 0.00 & & 0.13 & \\
$F$ for change in $R^{2}$ & & 0.06 & & 41.25 \\
\hline
\end{tabular}

${ }^{*} p<0.05 ; * * p<0.01 ; * * * p<0.001$.

impact on the cutaneous body image. $R^{2}=0.13$ meaning that the Model 2 explains $13 \%$ of the variance, $F(1,250)=21.21, p=0.000$ (Table 6$)$.

\section{Qualitative Analysis}

Thematic context analysis shows that the perceived impact of social distancing on skin health is pivotal. Emerging themes include the trigger effect of emotions on skin symptoms, the interdependence of environmental changes on skin symptoms, and the critical role of everyday life changes on skin symptoms ( $c f$. Table 7).

Main results show that besides the presence of a diagnosed skin disease $(N=89)$, half of the sample $(N=125)$ exhibited skin symptoms such as red rashes, eczema, or dry skin.

The majority of participants $(n=78)$ refer to stress and anxiety as a determinant factor of all skin symptoms. Participants explain the different sources of perceived stress: "Due to stress and anxiety about the financial outcome of this crisis" (44-year-old woman with acne).

"Nervousness that leads to scratching" (woman, 30 with urticaria).

Perceived fear linked to the COVID-19 pandemic is also a trigger for skin manifestations: "[I guess I have these skin symptoms] because of psychological

Table 7 Themes and categories of the perceived impact of social distancing on participants with dermatological symptoms (with or without a skin diagnosis)

\begin{tabular}{ll}
\hline Theme & Category \\
\hline Emotions triggering skin symptoms & $\begin{array}{l}\text { Stress due to COVID-19 } \\
\text { Fear due to COVID-19 } \\
\text { Boredom of staying inside } \\
\text { Environmental alterations }\end{array}$ \\
Reduced sun exposure \\
Lack of fresh air \\
Reduced pollution exposure \\
Sleep/Nutrition disorder \\
Temporal disorientation \\
Limited physical activity \\
Frequent use of hand sanitizers \\
Reduced skincare
\end{tabular}


pressure induced by the confinement and fear of what is going to happen next" (24-year-old woman with dry skin).

"Stress and fear about the COVID situation, has a negative effect on my acne" (23-year-old man with acne).

Some participants mention that they touch and scratch themselves more often because they are currently feeling bored: "I guess I touch and "play" with my face without noticing because I'm bored" (21-year-old woman with acne). "Mostly the lack of activities urges me to just move my hands all the time and I just can't do anything else [but touch my skin]" (19-year-old man with acne and urticaria).

As far as environmental alterations are concerned, participants state that reduced sun exposure along with the lack of fresh air and reduced pollution exposure are some of the reasons that affect their skin symptoms. "Only lack of sun exposure has an impact" (23-year-old woman with acne). On the other hand, two participants with acne state that reduced exposure to air pollution has had a positive impact: "There is no impact. In contrast, my acne reduces because I don't put daily make up on and I don't have exposure to air pollution" (24-yearold woman with acne).

Lockdown and social distancing lead to core changes to everyday life routine: Sleep, nutrition disorder, and limited physical activity are some of the most common answers related to everyday life changes: "It's all about stress, bad sleep and changes in daily schedule(nutrition, workout)" (22-year-old woman with acne).

The stay home policy leaded some of the participants to reduced skincare and a feeling of "letting oneself go": "I care less about myself. I don't buy the right creams because they are expensive" (25-year-old woman with eczema). Others state that "longer use of the same clothes while staying home" (20-yearold woman with urticaria) could actually worsen skin symptoms. Along the same lines, a certain form of disorientation also occurred during the lockdown period: "I feel like my ability to concentrate has dramatically dropped; being disoriented timewise" (32-year-old woman with multiple dermatological symptoms).

Frequent use of hand sanitizers or frequent hand washing seems to be another important factor triggering eczema or other skin symptoms such as dry skin. "Frequent handwashing resulted in my hand eczema" (44-year-old man with eczema). Nevertheless, acne patients did not mention this factor as a trigger to their acne symptoms.

\section{Discussion}

The aim of this study was the investigation of perceived stress, body image, and cutaneous body image on 253 young adults with or without skin diseases and manifestations. 


\section{Dermatological Manifestation and Control Group About Stress and Body Image}

In the current study, there are no significant differences in perceived stress between study and control groups apart from the sense of self-efficacy dimension. Our results contradict with those of Cash (2004) and Mikołajczyk et al. (2017) who support that individuals with dermatological diseases experience higher stress levels. We may hypothesize that the current stressful pandemic and social distancing measures consist of a major stressor in people's lives and the diagnosis of a dermatological disease does not have any consequences on stress levels (Taylor et al., 2008). Disease visibility seems to hold sway over the sense of selfefficacy as a perceived stress dimension (Dalgard et al., 2012). Surprisingly, in our sample, participants who suffer from acne experience a higher sense of selfefficacy while comparing to those who suffer from another dermatological disease. Papadopoulos, Walker, Aitken, and Bor (2000), in their research on a sample of 82 participants with acne and 107 without acne, revealed similar results, according to which participants with acne had a lower sense of self-efficacy than the control group.

The individuals who took part in our sample are generally satisfied with their overall body image; women placing a higher premium on their appearance than men. These results are in agreement with existing literature, where the MBSRQ scale was used (Argyrides and Kkeli, 2013; Muth \& Cash, 1997). In contrast to our hypothesis, the control group presenting dermatological diseases does not seem to have higher levels of body image satisfaction as expected. This result contradicts with previous research in which individuals confronted with skin diseases tend to undervalue their body image (Granström et al., 2012; Gündüz et al., 2020). However, previous studies have used different instruments to evaluate body image perception. More specifically, in the academic publication of Gündüz et al. (2020), the Body Cathexis Scale (BCS) was used, which determines the degree of self-evaluation of an individual's own feelings towards various body parts or functions (Secord \& Jourard, 1953), whereas research conducted by Granström et al. (2012) employed "insecurity/uneasiness" and "attractiveness/ self-confidence," which were adopted from the German edition of "Evaluation of the Own Body Questionnaire," a multidimensional self-report tool with 52 items and dichotomized response options.

In our sample, overweight preoccupation declines when cutaneous body image satisfaction rises, and it increases when participants score higher levels of "selfclassified weight." This result may prompt speculation about whether individuals who invest more in their respective cutaneous body image and are therefore more satisfied by it will be less preoccupied with overall body image. In terms of selfclassified weight, individuals who are more preoccupied with their weight tend to focus on their weight and being dissatisfied.

No significant differences could be seen between men and women, as the lockdown appears to be equally stressful for both genders. Statistical differences were only identified in terms of the appearance orientation subscale and cutaneous body image scale, which could be attributed to the Western ideal (Mikołajczyk, et al., 2017). Flawless skin ideals and cutaneous body image evaluation seem to 
be dominant among women in our sample, confirming our hypothesis. In similar research papers (Rumsey et al., 2004), findings emphasize the strict evaluation that women would make of their own cutaneous appearance. However, qualitative analysis highlights that participants believe the lockdown has a positive outcome on their skin disease, especially on acne, due to the reduced sun and pollution exposure as well as the reduced use of cosmetics.

The qualitative analysis further highlights the perceived factors that trigger skin symptoms for the participants of our sample such as stress about the outcomes of this pandemic, fear of the unknown regarding the lockdown restrictions imposed by the Greek government, along with changes in every aspect of the participants' lives. Stress and fear directly linked to this health crisis have already been discussed in existing literature (Asim et al., 2020; Horesh \& Brown, 2020; Shigemura et al., 2020) and since both of them are highly associated to skin symptoms and manifestations, participants are well aware of the reasons that triggered them. However, in terms of everyday routine alterations, research has shown that exposure to stressors is highly correlated to binge eating and a general drive to eat, which is deemed to be a contributing factor of excessive weight gain, affecting mostly women (Groesz et al., 2012).

There are potentially additional factors, which have a crucial role, such as uncertainty surrounding COVID-19, concerns about what the future may hold, and stress levels escalating as a result of following COVID-19-related news, updates, and rumors (Taylor et al., 2008). Additionally, the COVID-19 outbreak might have directed participants' attention to the ongoing pandemic and the repercussions it may have on their lives instead of their appearance. Nonetheless, multiple factors should be taken into consideration such as the aspect of self-compassion: according to recent academic studies (Linardon et al., 2020), individuals who lack selfcompassion tend to form a more negative evaluation of their body image. In their research, based on a sample comprising 992 adults, Linardon et al. (2020) suggest that self-compassion has a strong influence on someone's perception of their own body image dimension of form and weight evaluation.

\section{Limitations}

The current sample is not fully representative due to online recruiting. Our sample is quite heterogeneous which leads to a multitude of results but, simultaneously, it is difficult to extrapolate results across larger population groups. For instance, solely $17.4 \%$ of the sample is diagnosed with acne. For this reason, our results cannot be generalized whereas causal relationships cannot be established.

From a methodology perspective, a qualitative approach will be appropriate in order to explore in depth someone's very own experiences about their cutaneous body image and perceptions about their skin symptoms.

Our research was conducted amid the Greek lockdown, so a longitudinal approach will be useful to investigate how those surveyed evaluate the increase in dermatological symptoms and the perceived stress in the long term. 


\section{Future Research and Implications}

The findings of the current study explore significant aspects of perceived stress, body image, and cutaneous body image because dermatological disorders like acne, eczema, and urticaria have a bidirectional effect on patients. Even though our sample mostly consisted of young adults, including more adolescents in our sample could help us gain an even better insight, since the latter are more susceptible with regard to their body image along with their perceptions about their skin and overall appearance. Another interesting subject to explore extensively is the urticaria skin disease and how it may affect cutaneous body image and general body image since limited literature has been publicized in this field.

Moreover, patients with severe dermatological manifestations experience a type of rejection through the lack of dermatological contact. Conducting research on whether there are differences between the study and control groups, as dermatological contact is absent in both groups due to social distancing, could provide compelling findings. Finally, future studies will need to investigate protective factors, which can help a person to maintain his/her skin symptoms at a low level even during crisis situations. Last but not least, further research is required in order to gain a better understanding of potential gender differences, as encountered in a lockdown.

\section{Conclusion}

Overall, our findings emphasize that perceived stress is high during the lockdown period due to COVID-19 and it is considered one of the main skin symptom triggers whether there is a diagnosed skin disease or not. Taking into account that the genesis of a pandemic and the imposition of a mandatory lockdown provoke severely stressful reactions (ambiguity surrounding the duration of this health crisis, fear about what the future may hold, and secondary effects such as financial struggles) can certainly help elucidate its mediating role. Nonetheless, further research regarding this new norm is required to help us gain a better understanding and draw conclusions with regard to its effects from a psychological perspective. Thus, supporting individuals with dermatological symptoms to increase their cutaneous body image and raise awareness to individuals without skin diseases seems essential. This study can also contribute to the proposal for interventions with respect to patients who suffer from dermatological diseases. Different types of interventions should be recommended in order to broaden our knowledge, especially for younger age groups when the first dermatological symptoms occur. One of them could be to facilitate training programs in high schools aiming to raise awareness about skin diseases and their psychosomatic nature, along with the impact they may have on the body image perception, seeking improvement of general body and cutaneous image.

Finally, these results could be adopted to prevent social stigma and discrimination about certain dermatological diseases such as psoriasis or urticarial, which appear to be less common than others (e.g., acne). 
Acknowledgements A special thanks to every individual that voluntarily agreed to participate in our research during the challenging period of the national lockdown in Greece.

We would like to thank Mr. Argyris Argyroudis for reviewing and correcting this paper.

Data Availability Statement The datasets generated during and/or analyzed during the current study are available from the corresponding author on reasonable request.

\section{Declarations}

Ethical Statement Approval was obtained from the ethics committee of the National and Kapodistrian University of Athens. The procedures used in this study adhere to the tenets of the Declaration of Helsinki.

Conflict of Interest The authors have no relevant financial or non-financial interests to disclose.

Informed Consent Statement All participants were informed of the study's objectives and they completed a consent sheet beforehand.

\section{References}

Amr, M, Kaliyadan, F., \& Shams, T. (2014). Use of a Cutaneous Body Image (CBI) scale to evaluate self perception of body image in acne vulgaris. Acta Dermatovenerologica Croatia, 22(3), 196-199.

Andreou, E., Alexopoulos, E. C., Lionis, C., Varvogli, L., Gnardellis, C., Chrousos, G. P., \& Darviri, C. (2011). Perceived stress scale: Reliability and validity study in Greece. International Journal of Environmental Research and Public Health, 8(8), 3287-3298. https://doi.org/10.3390/ijerph8083287

Anzieu, D. (1995). Le Moi-peau (1985). Éditions Dunod.

Argyrides, M., \& Kkeli, N. (2013). Multidimensional body-self relations questionnaire-appearance scales: Psychometric properties of the Greek version. Psychological Reports, 113(3), 885-897. https://doi.org/10.2466/03.07.pr0.113x29z6

Asim, M., van Teijlingen, E., \& Sathian, B. (2020). Coronavirus Disease (COVID-19) and the risk of Post-Traumatic Stress Disorder: A mental health concern in Nepal. Nepal Journal of Epidemiology, 10(2), 841-844. https://doi.org/10.3126/nje.v10i2.29761

Beaton, D. E., Bombardier, C., Guillemin, F., \& Ferraz, M. B. (2000). Guidelines for the process of crosscultural adaptation of self-report measures. Spine, 25(24), 3186-3191.

Boehncke, W. H., \& Schön, M. P. (2015). Psoriasis. The Lancet, 386(9997), 983-994. https://doi.org/10. 1016/s0140-6736(14)61909-7

Bolognia, J. L., Schaffer, J. V., Duncan, K. O., \& Ko, C. J. (2014). Dermatology essentials E-book. Elsevier Health Sciences.

Bowe, W. P., Doyle, A. K., Crerand, C. E., Margolis, D. J., \& Shalita, A. R. (2011). Body image disturbance in patients with acne vulgaris. The Journal of Clinical and Aesthetic Dermatology, 4(7), 35.

Braun, V., \& Clarke, V. (2006). Using thematic analysis in psychology. Qualitative Research in Psychology, 3(2), 77-101.

Cash, T. F. (2002). The situational inventory of body-image dysphoria: Psychometric evidence and development of a short form. International Journal of Eating Disorders, 32(3), 362-366.

Cash, T. F. (2004). Body image: Past, present, and future.

Cash, T. F., Santos, M. T., \& Williams, E. F. (2005). Coping with body-image threats and challenges: Validation of the Body Image Coping Strategies Inventory. Journal of psychosomatic research, 58(2), 190-199.

Cleary, M., Kornhaber, R., Thapa, D. K., West, S., \& Visentin, D. (2020). A quantitative systematic review assessing the impact of burn injuries on body image. Body Image, 33, 47-65.

Cohen, S., Kamarck, T., \& Mermelstein, R. (1983). A global measure of perceived stress. Journal of health and social behavior, 385-396.

Dalgard, F., Stern, R., Lien, L., \& Hauser, S. (2012). Itch, stress and self-efficacy among 18-year-old boys and girls: A Norwegian population-based cross-sectional study. Acta Dermato Venereologica, 92(5), 547-552. https://doi.org/10.2340/00015555-1309 
Georgiadis, G. S., Argyriou, C., Georgakarakos, E. I., \& Lazarides, M. K. (2021). Unmasking peripheral arterial disease in diabetic patients presenting with inflammatory skin manifestations during the COVID-19 pandemic. The International Journal of Lower Extremity Wounds, 15347346211004029.

Granström, S., Langenbruch, A., Augustin, M., \& Mautner, V. F. (2012). Psychological burden in adult neurofibromatosis type 1 patients: Impact of disease visibility on body image. Dermatology, 224(2), 160-167. https://doi.org/10.1159/000337548 Epub 2012 Apr 12 PMID: 22508340.

Groesz, L. M., McCoy, S., Carl, J., Saslow, L., Stewart, J., Adler, N., ... \& Epel, E. (2012). What is eating you? Stress and the drive to eat. Appetite, 58(2), 717-721

Grogan, S. (2017). Body image: Understanding body dissatisfaction in men, women and children. Routledge.

Gündüz, A., Topçuoğlu, V., Usta Gündüz, E. B., Ergun, T., Gençosmanoğlu, D. S., \& Sungur, M. Z. (2020). Significant effects of body image on sexual functions and satisfaction in psoriasis patients. Journal of Sex \& Marital Therapy, 46(2), 160-169.

Gupta, M. A., Gupta, A. K., \& Johnson, A. M. (2004). Cutaneous body image: Empirical validation of a dermatologic construct. Journal of Investigative Dermatology, 123(2), 405-406. https://doi.org/10. 1111/j.0022-202x.2004.23214.x

Gupta, M. A., \& Gupta, A. K. (2013). Evaluation of cutaneous body image dissatisfaction in the dermatology patient. Clinics in dermatology, 31(1), 72-79.

Hersey, P., Blanchard, K. H., \& Johnson, D. E. (2007). Management of organizational behavior (Vol. 9). Upper Saddle River, NJ: Prentice hall.

Horesh, D., \& Brown, A. D. (2020). Traumatic stress in the age of COVID-19: A call to close critical gaps and adapt to new realities. Psychological Trauma: Theory, Research, Practice, and Policy, $12(4), 331$.

Hosoi, J. (2006). Stress and the skin. International Journal of Cosmetic Science, 28(4), 243-246.

Johnson, R. B., Onwuegbuzie, A. J., \& Turner, L. A. (2007). Toward a definition of mixed methods research. Journal of Mixed Methods Research, 1(2), 112-133.

Lazarus, R. S., \& Folkman, S. (1984). Stress, appraisal and coping. Springer-Verlag.

Lee, E. H. (2012). Review of the psychometric evidence of the perceived stress scale. Asian Nursing Research, 6(4), 121-127.

Linardon, J., Susanto, L., Tepper, H., \& Fuller-Tyszkiewicz, M. (2020). Self-compassion as a moderator of the relationships between shape and weight overvaluation and eating disorder psychopathology, psychosocial impairment, and psychological distress. Body Image, 33, 183-189. https://doi.org/10. 1016/j.bodyim.2020.03.001

Mikołajczyk, J., Rzepa, T., Król, J., \& Żaba, R. (2017). Body image assessment and quality of life in patients with atopic dermatitis. Dermatology Review/Przeglad Dermatologiczny, 104(2).

Muth, J. L., \& Cash, T. F. (1997). Body-image attitudes: What difference does gender make? Journal of Applied Social Psychology, 27(16), 1438-1452. https://doi.org/10.1111/j.1559-1816.1997.tb01607.x

Papadopoulos, L., Walker, C., Aitken, D., \& Bor, R. (2000). The relationship between body location and psychological morbidity in individuals with acne vulgaris. Psychology, health \& medicine, 5(4), 431-438.

Parisi, R., Symmons, D. P. M., Griffiths, C. E. M., \& Ashcroft, D. M. (2013). Global epidemiology of psoriasis: A systematic review of incidence and prevalence. Journal of Investigative Dermatology, 133(2), 377-385. https://doi.org/10.1038/jid.2012.339

Partridge, J. (1993). The psychological effects of facial disfigurement. Journal of Wound Care, 2(3), 168-171.

Petersen, A. C., Schulenberg, J. E., Abramowitz, R. H., Offer, D., \& Jarcho, H. D. (1984). A self-image questionnaire for young adolescents (SIQYA): Reliability and validity studies. Journal of Youth and Adolescence, 13(2), 93-111.

Peterson, C. (2010). Learned helplessness. The Corsini Encyclopedia of Psychology. https://doi.org/10. 1002/9780470479216.corpsy0500

Roy, M., \& Payette, H. (2012). The body image construct among Western seniors: A systematic review of the literature. Archives of Gerontology and Geriatrics, 55(3), 505-521.

Rumsey, N., Clarke, A., White, P., Wyn-Williams, M., \& Garlick, W. (2004). Altered body image: Appearance-related concerns of people with visible disfigurement. Journal of Advanced Nursing, 48(5), 443-453. https://doi.org/10.1111/j.1365-2648.2004.03227.x

Sadeghzadeh-Bazargan, A., Rezai, M., Nobari, N. N., Mozafarpoor, S., \& Goodarzi, A. (2021). Skin manifestations as potential symptoms of diffuse vascular injury in critical COVID-19 patients. Journal of cutaneous pathology 
Saif, G. A. B., Alotaibi, H. M., Alzolibani, A. A., Almodihesh, N. A., Albraidi, H. F., Alotaibi, N. M., \& Yosipovitch, G. (2018). Association of psychological stress with skin symptoms among medical students. Saudi Medical Journal, 39(1), 59.

Salari, N., Hosseinian-Far, A., Jalali, R., Vaisi-Raygani, A., Rasoulpoor, S., Mohammadi, M., ... \& KhalediPaveh, B. (2020). Prevalence of stress, anxiety, depression among the general population during the COVID-19 pandemic: A systematic review and meta-analysis. Globalization and health, 16(1), 1-11

Schacter, L. D., Gilbert, T. D. \& Wegner, M. D. (2012). Psychology (2nd Edition). New York: Worth; 2011.... Psychology (2nd Edition).

Secord, P. F., \& Jourard, S. M. (1953). The appraisal of body-cathexis: body-cathexis and the self. Journal of consulting psychology, 17(5), 343.

Schoonenboom, J., \& Johnson, R. B. (2017). How to construct a mixed methods research design. KZfSS Kölner Zeitschrift Für Soziologie Und Sozialpsychologie, 69(2), 107-131.

Shigemura, J., Ursano, R. J., Morganstein, J. C., Kurosawa, M., \& Benedek, D. M. (2020). Public responses to the novel 2019 coronavirus (2019-nCoV) in Japan: Mental health consequences and target populations. Psychiatry and clinical neurosciences

Simsek, N., Evli, M., Uzdil, N., Albayrak, E., \& Kartal, D. (2020). Body image and sexual self-confidence in patients with chronic urticaria. Sexuality and Disability, 1-13

Stalikas, A., Triliva, S., \& Roussi, P. (2012). Psychometric tools in Greece. Athens, Greece: Pedio.

Stefanaki, C., Stratigos, A. J., \& Stratigos, J. D. (2002). Skin manifestations of HIV-1 infection in children. Clinics in Dermatology, 20(1), 74-86.

Taylor, M. R., Agho, K. E., Stevens, G. J., \& Raphael, B. (2008). Factors influencing psychological distress during a disease epidemic: Data from Australia's first outbreak of equine influenza. BMC Public Health, 8(1), 347.

Taylor, S., Landry, C. A., Paluszek, M. M., Fergus, T. A., McKay, D., \& Asmundson, G. J. G. (2020). Development and initial validation of the COVID Stress Scales. Journal of Anxiety Disorders, 72, 102232. https://doi.org/10.1016/j.janxdis.2020.102232

Thompson, J. K. (2004). The (mis) measurement of body image: Ten strategies to improve assessment for applied and research purposes. Body Image, 1(1), 7-14.

Thompson, J. K., \& Schaefer, L. M. (2019). Thomas F. Cash: A multidimensional innovator in the measurement of body image; Some lessons learned and some lessons for the future of the field. Body Image, 31, 198-203.

Warren, C. S., Holland, S., Billings, H., \& Parker, A. (2012). The relationships between fat talk, body dissatisfaction, and drive for thinness: Perceived stress as a moderator. Body Image, 9(3), 358-364. https://doi.org/10.1016/j.bodyim.2012.03.008

Zandifar, A., \& Badrfam, R. (2020). Iranian mental health during the COVID-19 epidemic. Asian journal of psychiatry, 51 .

\section{Authors and Affiliations}

\section{Sofia Aperi ${ }^{1} \cdot$ Flora Koliouli $^{2} \mathbb{D}$}

Sofia Aperi

sofiaaperi@gmail.com

1 Psychology Department, School of Philosophy, National and Kapodistrian University of Athens, Athens, Greece

2 Laboratory of Qualitative Research in Psychology and Mental Health, Department of Psychology, School of Philosophy, National and Kapodistrian University of Athens, Athens, Greece 